\title{
Modelling and Performance Evaluation of WiMAX 700 to Work as Secondary User in Spectrum of the Digital Television Broadcasting Terrestrial through the Cognitive Radio Network
}

\author{
Albedeir Y. Othman, Khald H. Belal, and Mohammed A. Elmaleeh
}

\begin{abstract}
The cognitive radio network (CRN) is considered as one of the most important technologies that can allow the different users work as the same spectrum by different priorities. This paper proposes new methods and solutions to implement and performance analysis of work the WiMAX system within the spectrum of digital television broadcasting terrestrial through the cognitive radio network under various conditioning and types of modulation. Also, this paper explains and analyze the mechanism in which the cognitive radio works. Finally will be designed a prototype device simulate work the WiMAX system as secondary user in digital TV broadcasting through the cognitive radio techniques.

Index Terms - Adaptive Modulation and Coding, Bit Error Rate, Cyclic Prefix, Cognitive Radio Networks, Digital Television, Digital Video Broadcasting -Terrestrial, Primary User, Signal-to-Noise Ratio, Secondary User, Receiver Operating Characteristic, WiMAX system.
\end{abstract}

\section{INTRODUCTION}

The main idea for this paper is creating a new method to utilize the (White Spaces ) resulting from transformation the television broadcasting terrestrial systems from the analog to digital system Within the frequency spectrum range Very high frequency (VHF) and Ultra High Frequency (UHF) and to benefit from the relatively large guard band in the TV signal and benefit from the early detection to Borrowing frequency If necessary, The main idea is mainly based on a TVCRW adaptation (Television-Cognitive Radio-WiMAX) which is the management and control part of the complete system and consists of three main units: the radio-sensing unit, the storage unit, the account and logic unit ,These modules were enhanced by the neural network algorithm and a new algorithm that was created (the sorting algorithm or Bedeir's algorithm).

This three Units are designed and programmed to operate between two different integrated systems, in the operating priorities and mechanisms. The basic system (primary user) is the digital television broadcasting terrestrial system and the secondary user is the WIMAX system. The broadcasting station provides full spectrum which is based on the orthogonal theory for the basic system, as for the secondary system uses the same frequency spectrum by means of certain mechanisms and methods that are derived from the TVCRW in three cases:
1. Exploitation of the separation areas in radio spectrum of the basic system after ensuring the compatibility of the channel and the WiMAX signal to be sent and its approval of the channel conditions.

2. Availability of the channel and not occupied by the primary user (basic system).

3. Exploitation of another frequency that is already known to the appropriate TVCRW.

In the beginning, the two systems are aligned to work on the same criterion and standard conditions as are compatible with the $4 \mathrm{G}$ standard and to configure the channel to operate under these standards and to adapt them to deal with variables input while maintaining the constants and limitations of broadcasting. The frequency spectrum is then exploited by the DVB-T system. The TVCRW adaptor works on adjusts the signal changes, signal-to-noise ratio, the associated changes and the power changes, and then make math's calculations and logical calculations and then make sure that the channel is available to be used by the secondary system or not under the instruction of the cognitive radio unit in the secondary system. If the channel is occupied by the primary system, the data of secondary system is sent which was previously preparation by means of a modified triple repeat for the data, and then stored in three special registers. These registers correspond to the processing method to be followed in the transmission, which is selected according to the outputs of the TVCRW adapters and is called by the management and control unit located in the system in the TVCRW, after that the signals of the secondary user is sent within the same ether with the primary user, both packets of the two systems are received across the antenna in the form of frequency bands within the spectrum and are processed via added algorithms for all processing units at each repeat station. The Interrupt system is activated if the primary user needs to use the channels and the channel was occupied by the secondary system. And the signal for the secondary user is compensated from one of the three registers and sent according to the directions of TVCRW adapter.

\section{ISSUES}

Implement a system capable of enabling the different users to benefit from the best television services and broadband services through the use of digital terrestrial broadcasting without interference between the two services. And increased the bandwidth for broadband services to include additional 
geographic areas that contribute to lower costs and equipment. Keeping abreast of developments and providing additional options for services that did not exist such as interactive services and others. And improve some important parameters of the WiMAX system such as error bit rate and increase the efficiency of receptors and increase the rate of data transmission with the ability to control and save the power at a suitable value. Providing a clearer and clearer picture using digital television broadcasting as an alternative to analog broadcasting.

The settings used in the simulation program are shown in table 1. All results in this paper for the modulation schemes and coding rates have been obtained in an AWGN channel and with the average of SNR is 18. And used the six different modulations order and coding rates for the purpose of data transmission. They are shown in table 2.

\section{MATERIALS AND METHODS}

This research has been studied in two parts: the first part is the simulation through using the Matlab program, The second part is the practical design of an integrated system consisting of programmable controllers (Raspberry Pi and Arduino), transmitters and receivers (RF433MHZ) and other accessories (input and output devices).in simulation and practical model the WiMAX system represents a secondary user and the DVB-T represent the DTV or the primary user, and the frequency detection mechanism used in this model is the energy detection method.

\begin{tabular}{|c|c|c|c|}
\hline $\begin{array}{c}\text { Channel bandwidth } \\
\text { (MHz): }\end{array}$ & $\begin{array}{l}7 \mathrm{MHz}: \text { same } \\
\text { as TV channels } \\
\text { (according to } \\
\text { regulatory } \\
\text { domain) } \\
\end{array}$ & $\begin{array}{l}\text { Number of tests } \\
\text { performed: }\end{array}$ & 11 cases \\
\hline $\begin{array}{l}\text { Number of OFDM } \\
\text { symbols per burst: }\end{array}$ & 1,2 & Simulation mode: & Monte -carlo \\
\hline $\begin{array}{l}\text { Cyclic prefix factor } \\
\text { (G): }\end{array}$ & $1 / 4$ & Code rate $(\mathrm{CC})$ : & $1 / 2,2 / 3,3 / 4$ \\
\hline Additional Algorithms: & $\begin{array}{l}\text { neural networks } \\
\text { (NN), } \\
\text { sorting } \\
\text { algorithm (BA) } \\
\end{array}$ & $\begin{array}{l}\text { Detection } \\
\text { method: }\end{array}$ & $\begin{array}{l}\text { Energy of } \\
\text { signals }\end{array}$ \\
\hline Modulation: & $\begin{array}{l}\text { QPSK,16QAM, } \\
\text { 64QAM }\end{array}$ & Fading mode: & $\begin{array}{l}\text { frequency- } \\
\text { selective fading }\end{array}$ \\
\hline $\begin{array}{c}\text { Models a multipath } \\
\text { Rician fading channel } \\
\text { with : } \\
\end{array}$ & AWGN & Rate ID without: & $1-6$ \\
\hline Number of test rounds: & 30 rounds & Threshold mode: & Single tone \\
\hline
\end{tabular}

Table 2: Modulation Schemes and Coding rates.

\begin{tabular}{|c|c||}
\hline Rate-ID & Modulation RS-CC rate \\
\hline \hline $\mathbf{1}$ & QPSK 1/2 \\
\hline $\mathbf{2}$ & QPSK 3/4 \\
\hline $\mathbf{3}$ & 16-QAM 1/2 \\
\hline \hline $\mathbf{4}$ & 16-QAM 3/4 \\
\hline $\mathbf{5}$ & 64-QAM 2/3 \\
\hline \hline 6 & $64-Q A M ~ 3 / 4$ \\
\hline
\end{tabular}

\section{Probability and Hypothesis Testing Equations}

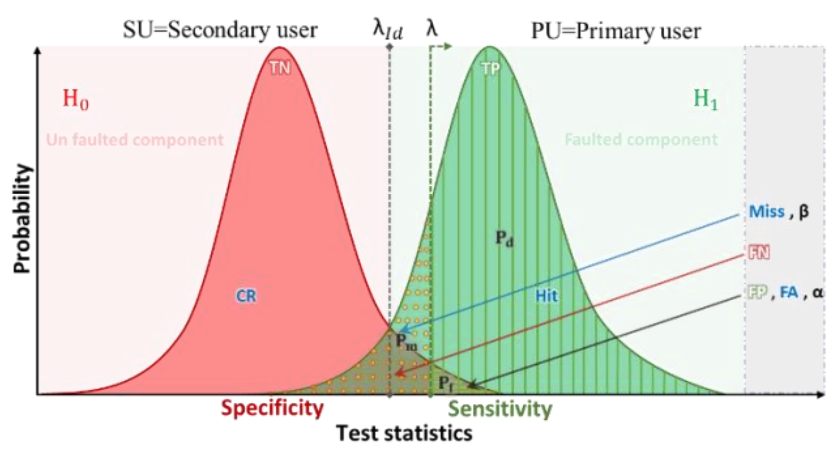

\begin{tabular}{|c|c|}
\hline $\mathbf{H}_{1}$ : Alternate hypothesis & $\mathbf{P}_{\mathrm{f}}$ : Probability of false alarm \\
\hline $\mathrm{H}_{0}$ : Null hypothesis & $\mathbf{P}_{\mathrm{m}}$ : Probability of missed detect \\
\hline$\lambda$ : Alarm Threshold & TN : True Negative \\
\hline$\lambda_{l d}$ : Ideal observer's threshold & FN : False Negative \\
\hline CR : Correct Rejection & TP : True Positive \\
\hline FA : False alarm & FP : False Positive \\
\hline $\mathbf{P}_{\mathbf{d}}$ : Probability of detection & $\alpha:$ Represents $P_{\mathrm{f}}, \boldsymbol{\beta}:$ Represents $\mathrm{P}_{m}$ \\
\hline
\end{tabular}

Figure 1: Binary Hypothesis Testing.

Table 3: Sensitivity and Specificity.

\begin{tabular}{|c|c|c|}
\hline & $\boldsymbol{H}_{1}$ Signal present & $\boldsymbol{H}_{\mathbf{0}}$ Signalabsent \\
\hline Detection & True Positive & False Positive \\
\hline Null result & False Negative & True Negative \\
\hline Range indication & TPF $=$ Sensitivity & FPF = Specificity \\
& $\frac{\mathrm{TP}}{(\mathrm{TP}+\mathrm{FN})}$ & $\frac{\text { FP }}{(\mathrm{FP}+\mathrm{TN})}$ \\
\hline
\end{tabular}

Can use the energy detection to detect and analysis the signal by more methods but the most famous is hypothesis testing So that the receiving signal is represented by $y_{n}$ shown below equation 1 and 2.

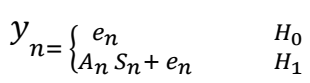

$$
\begin{aligned}
& \text { Energy Detection }=\left\{\begin{array}{lc}
H_{0}, \text { if } & \sum_{n=1}^{N}\left[y_{(n)}\right]^{2} \leq \lambda \\
H_{1}, & \text { Otherwise }
\end{array}\right.
\end{aligned}
$$

Where $\mathrm{H}_{1}$ is represent alternative hypothesis, $\mathrm{H}_{0}$ is represent null hypothesis, $A_{n}$ represent the gain and $S_{n}$ is the signal information and $e_{n}$ represent the noise and $\lambda$ is threshold. For calculating the probability percentage for the signals, can use the following equations no.3, 4 and 5 to calculate the probability of detection. Also equation no. 6 to calculate the probability of detection if a false-alarm probability is known. Equation no. 7 to calculate the probability of false alarm, and equation no. 8 to calculate the probability of missed detection.

$$
\begin{gathered}
P D=\frac{\text { Number of acquisitions }}{\text { Total number of opportunities }}=\frac{\text { Over_Num }}{K K} \\
P D=1-P_{M}=1-P\left(\frac{H_{0}}{H_{1}}\right)
\end{gathered}
$$




$$
\begin{gathered}
P D=\left[e^{\frac{-T h}{2}} * \frac{1}{n !}\left(\frac{T h}{2}\right)^{n}\right]+\left[e^{\frac{-T h}{2(1+L)}} *\left(\frac{1+L}{L}\right)\right]-\left[e^{\frac{-T h}{2}} * \frac{1}{n !} * \frac{T h * L}{2(1+L)}\right] \\
P D=\frac{1}{2} \operatorname{erfc}\left(\operatorname{erfc}^{-1}\left(2 P_{F A}\right)-\sqrt{x}\right)
\end{gathered}
$$

Where $P D$ represent the detection probability and Th is threshold and $\mathrm{L}$ is the signal to noise ratio and $P_{M}$ represent the Probability of missed detection $P_{F A}$ is the false-alarm probability, erfc and $e r f c^{-1}$ are the complementary error function and that function's inverse, and $\chi$ is the SNR not expressed in decibels.

$$
P F=P\left(\frac{H_{1}}{H_{0}}\right)=P\left(\frac{P U}{H_{0}}\right)=P\left(\frac{y_{n}}{H_{0}}\right)=1-F_{H_{0}}(T h)
$$

Where $\mathrm{PF}$ is the false alarm probability and $\mathrm{F}_{\mathrm{H}_{0}}$ represent the cumulative distribution function (CDF).

$$
P m=1-P D
$$

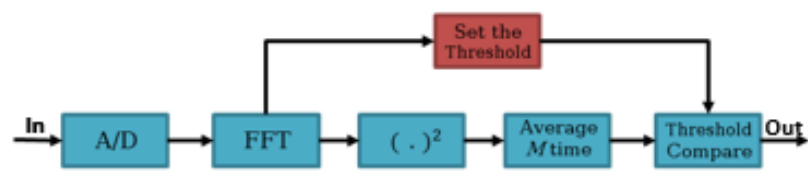

Figure 2: shows the architecture for digital implementation of an energy detector.

\section{RESULTS}

In the Matlab simulation the six cases were tested. So that each test represents a specific case as shown in the table4.

Table 4: Shows the simulation settings.

\begin{tabular}{||c||l||}
\hline Number of case & \multicolumn{1}{|c||}{ Content of the Test } \\
\hline \hline \multirow{5}{*}{ Case 1 } & $\begin{array}{l}\text { Probability of the primary user utilization } \\
\text { of frequency, Do not use the frequency by } \\
\text { any users, The frequency utilization by the } \\
\text { secondary user, The white frequency } \\
\text { exploitation by secondary user. } \\
\text { Exploitation of frequency reuse by the } \\
\text { secondary user, With the activation of } \\
\text { cognitive property. }\end{array}$ \\
\hline \hline \multirow{5}{*}{ Case 2 } & $\begin{array}{l}\text { Probability of the primary user utilization } \\
\text { of frequency, The frequency utilization by } \\
\text { the secondary user, The white frequency } \\
\text { exploitation by secondary user, with the } \\
\text { continuous activation of cognitive } \\
\text { property. }\end{array}$ \\
\hline \hline Case 3 & $\begin{array}{l}\text { Probability of the primary user utilization } \\
\text { of frequency, The frequency utilization by } \\
\text { the secondary user, With the continuous } \\
\text { activation of cognitive property. }\end{array}$ \\
\hline \hline
\end{tabular}

\begin{tabular}{|c|l||}
\hline Case 4 & $\begin{array}{l}\text { The frequency utilization by the secondary } \\
\text { user all the time. }\end{array}$ \\
\hline \hline Case 5 & $\begin{array}{l}\text { The frequency utilization by the primary } \\
\text { user all the time. }\end{array}$ \\
\hline \hline Case 6 & $\begin{array}{l}\text { Probability of the primary user utilization } \\
\text { of frequency, Do not use the frequency by } \\
\text { any users, The frequency utilization by the } \\
\text { secondary user, The white frequency } \\
\text { exploitation by secondary user. } \\
\text { Exploitation of frequency reuse by the } \\
\text { secondary user, With the activation of } \\
\text { cognitive property and different type of } \\
\text { modulation for the first case. }\end{array}$ \\
\hline
\end{tabular}

A. The results of the active window for the cognitive radio technique to allow the WiMAX system to exploit the frequencies in spectrum of DTV as an unlicensed user

The active window or the active area is represents the exploratory space in which the cognitive radio technique sensor operates by sensing the power of signals in the TV spectrum. And then identification of vacant frequencies and empty frequencies.To achieve this, many repetitive loops are conducted to make sure that the frequency to be used is not vacant ,according to several determinants of which the most important is the base of false alarm and the method used to detecting the signals and the algorithm which implemented for this purpose.

In this paper to the purpose of detecting and determining the signal is used in the simulation model the mechanism of the energy detection algorithm, and used the monte-carlo method which depend on repetition 1000 times before giving the result, And use the base false alarm by five levels from 0.1 to 1 with a range of 0.2 . To be effective the cognitive window and with a capacity range of $1000 * 5$ or 50000 times, That means the result does not depend on a critical one point , and also must be confirmed the final result by the many identical results. For this reason, launched the term "Effective Window" on this process.

All these precautions and procedures are done to ensure that the primary user is not confused or affected by any secondary unlicensed user. The figure 3 and 8 shows the active window for Cognitive Radio to control the frequencies in the TV spectrum according to the priorities granted to each user. Notes here that the channel is busy by the primary user, so the WiMAX system will operate on the white space if has found any frequency empty or follow the orders from algorithm of the TVCRW. The figure 4 and 5 shows the active window for Cognitive Radio to control the frequencies in the TV spectrum according to the priorities granted to each user. Notes here that the channel is not busy by the primary user, so the WiMAX system will operate in the same frequency of primary user. And the cognitive radio will remain alert to any actual presence by the primary user to isolate the secondary user and provide the full frequency for the primary user with find the alternative solutions to the secondary user according to instruction of the TVCRW algorithm. The figure 6 and 7 shows the work of both the primary and secondary user separately according to programming setting in simulation. 


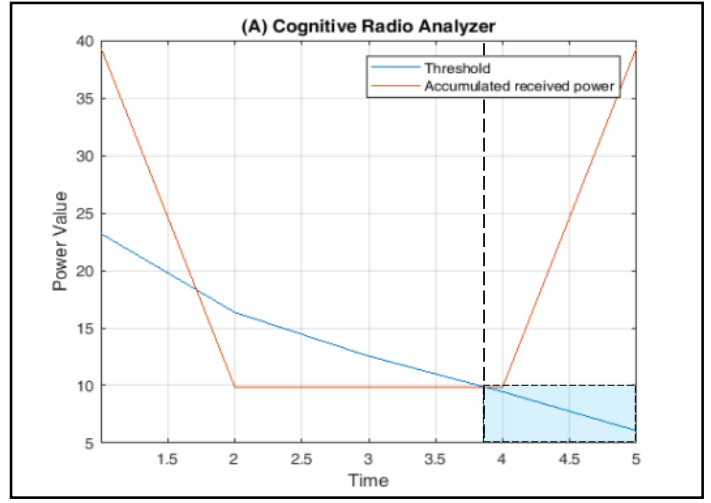

Figure 3: The active window for Cognitive Radio to control the white spaces under test case no. M1C1-A.

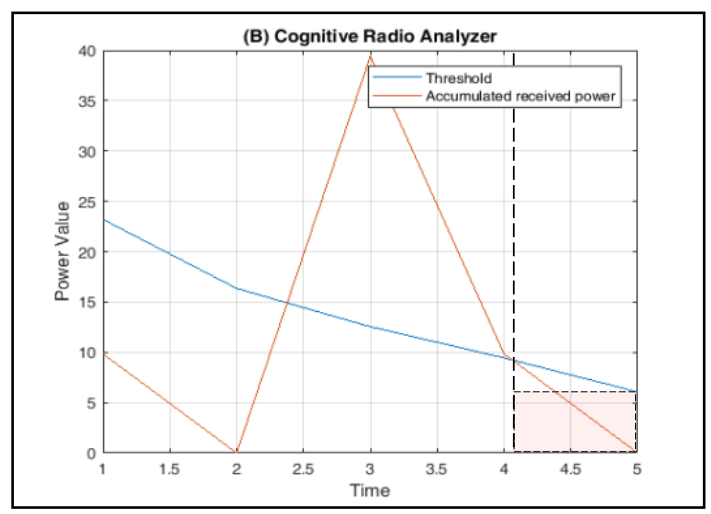

Figure 4: The active window for Cognitive Radio to control the white spaces under test case no. M1C1-B.

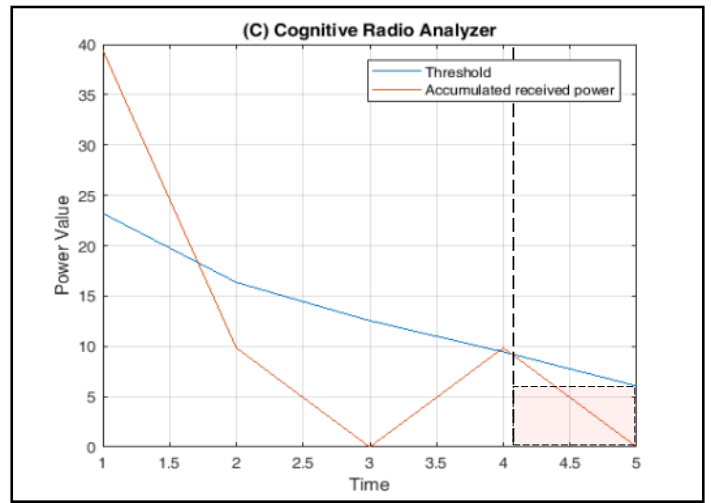

Figure 5: The active window for Cognitive Radio to control the white spaces under test case no. M1C1-C

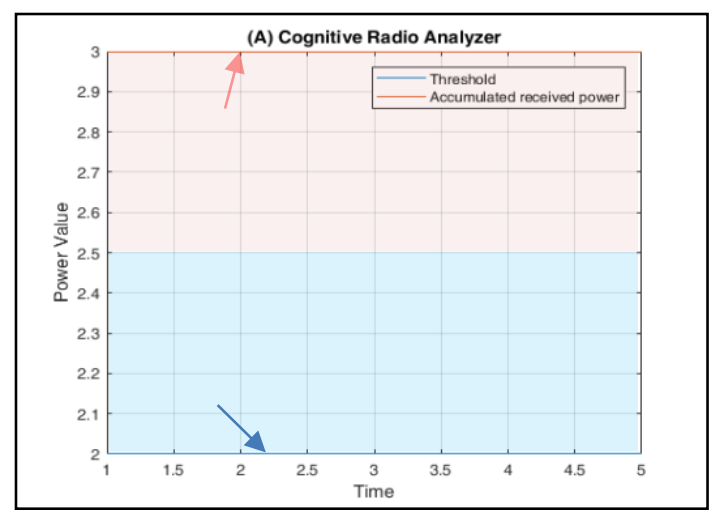

Figure 6: The active window for Cognitive Radio to control the white spaces under test case no. M1C3-A.

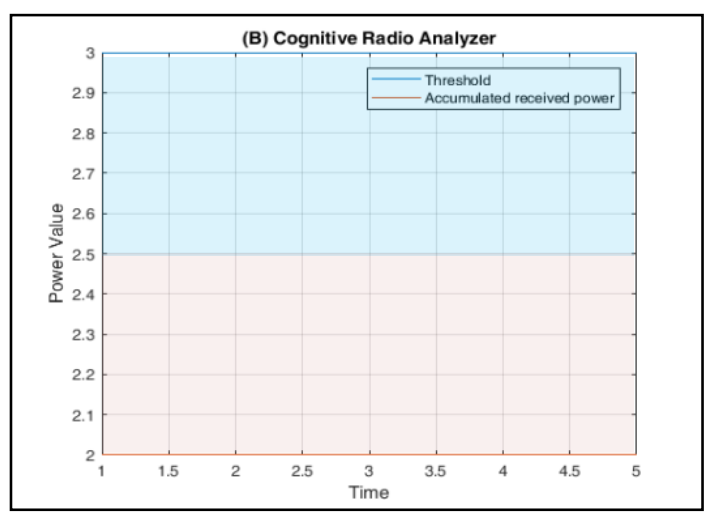

Figure7: The active window for Cognitive Radio to control the white spaces under test case no. M1C4-B.

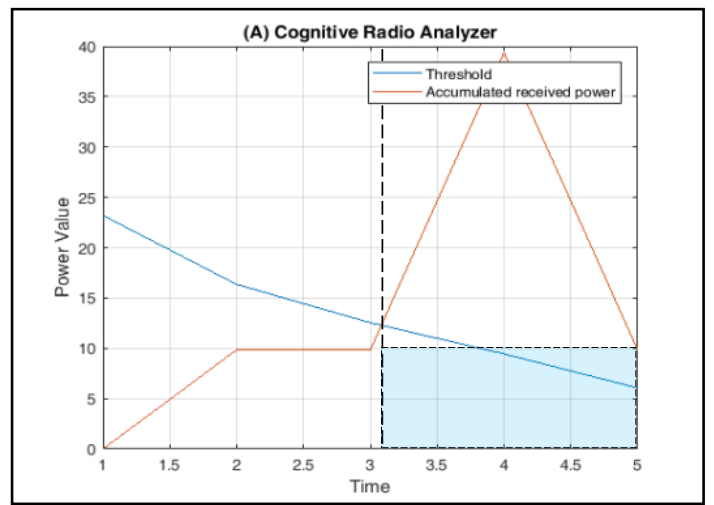

Figure 8: The active window for Cognitive Radio to control the white spaces under test case no. M1C6-A.

B. The results of the theoretical coherent receiver operating of energy detection for some types of probability with the different values of snr under AWGN.

Receiver Operating Characteristic (ROC) curves present graphical summaries of a detector's performance. The figure 9 shows the Theoretical Receiver Operating characteristic of energy detection under AWGN for the probability of false alarm versus the probability of detection when used the different values of signals to noise ratio (SNR). Where it is noted that the greater the probability of false alarm the greater the probability of detection and the opposite is true. From figure 9 the ROC curve in corresponding to an SNR of $7 \mathrm{~dB}$ by inspecting the graph with the data cursor, you can see that to achieve a probability of detection of 0.8 , you must tolerate a false alarm probability of up to 0.01 . The figure 10 shows the coherent Receiver Operating Characteristic to describe the theoretical relationship between SNR versus probability of detection to determine the probability of false alarm detection. The graph shows that, as the SNR increases, the supports of the probability distributions under the null and alternative hypotheses become more disjoint. Therefore, for a given false-alarm probability, the probability of detection increases. 


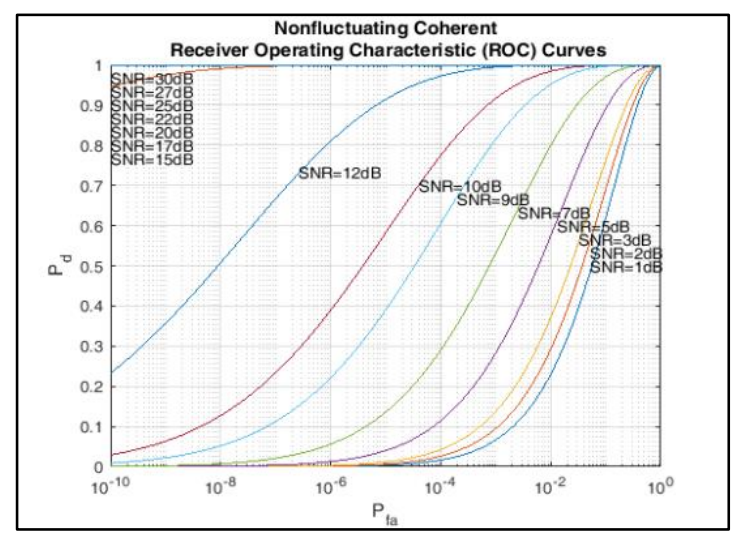

Figure 9: The nonfluctuating coherent ROC to describe the theoretical relationship between $\mathrm{Pd}$ vs Pf.

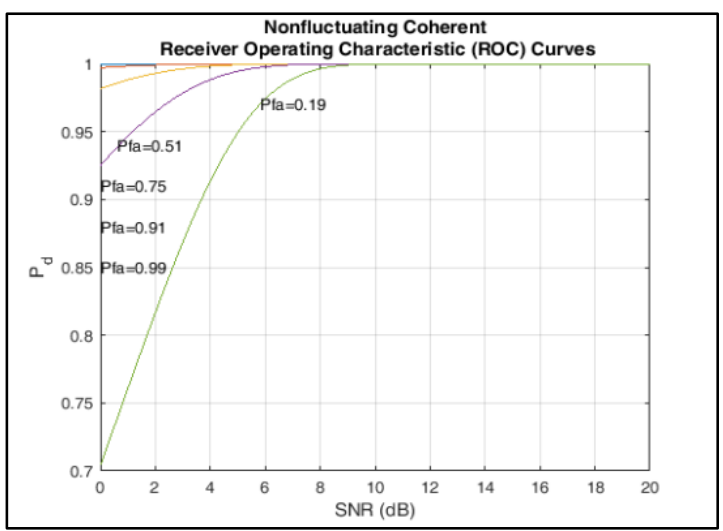

Figure 10: The nonfluctuating coherent ROC to describe the theoretical relationship between SNR vs Pd.

C. The results of complementary receiver operating characteristic of energy detection for all types of probability under AWGN channel.

The ROC graphs are a useful technique for organizing classifiers and visualizing their performance. ROC graphs are commonly used in engineering and medical field decision making. You can use this function only and only if you have a binary classificatory. To use the ROC must have two input is $\mathrm{Nx} 2$ matrix: in the first column you will put your test values and in the second column you will put only 1 or 0 (1 if the bad and 0 if good). The function computes and plots the classical ROC curve and curves for Sensitivity, Specificity and Efficiency.

The simulation includes calculating the threshold in energy detection. This is a general method and applicable to all scenarios for energy detection. All the signals are assumed to be complex and the algorithm is:

1) Assume only noise is received that means the primary user is absent.

2) If the only noise energy lies above the threshold, it corresponds to false alarm.

3) Run this scenario for some number of iteration.

4) $\mathrm{Pf}=$ energy above threshold / No. of Iteration.

The figure 11 shows the complementary Receiver Operating characteristic of energy detection under AWGN for the probability of false alarm versus the probability of detection. Where notes that the greater the probability of false alarm the greater the probability of detection and the opposite is true. The figure 12 shows the complementary Receiver Operating characteristic of energy detection under AWGN for the probability of detection versus the probability of missing. Where notes that the greater the probability of detection the decrease the probability of missing and the opposite is true. The figure 13 shows the probability of false alarm versus the probability of detection Where notes that the greater the probability of false alarm the probability of detection does not change from zero, because the simulation forced by both systems DTV \& WiMAX to work in the same spectrum but on the different channel all the time. The figure 14 shows nothing. That is result is normal because the signal cannot be detected from the previous result for the same reasons.

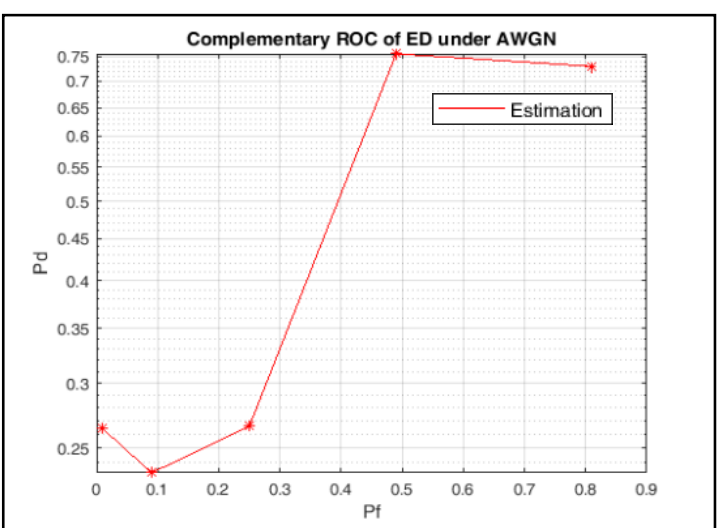

Figure 11: The complementary ROC of energy detection under AWGN for $\mathrm{Pf}$ vs Pd according test no. M1C1.

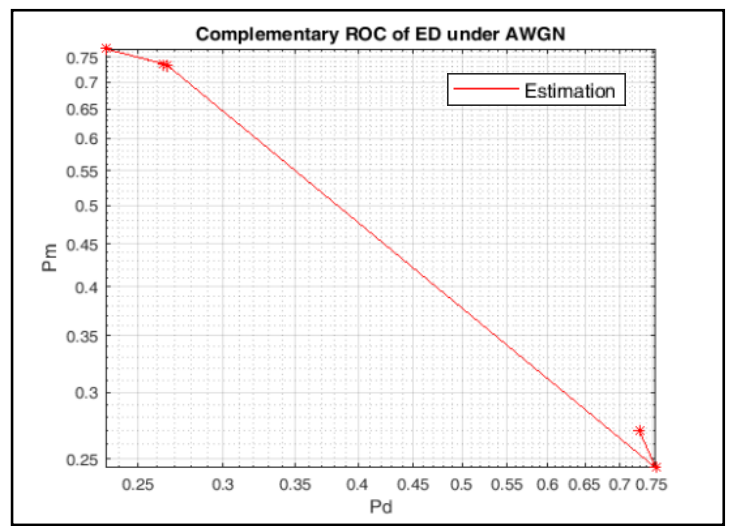

Figure 12: The complementary ROC of energy detection under AWGN for $\mathrm{Pd}$ vs Pm according test no. M1C1.

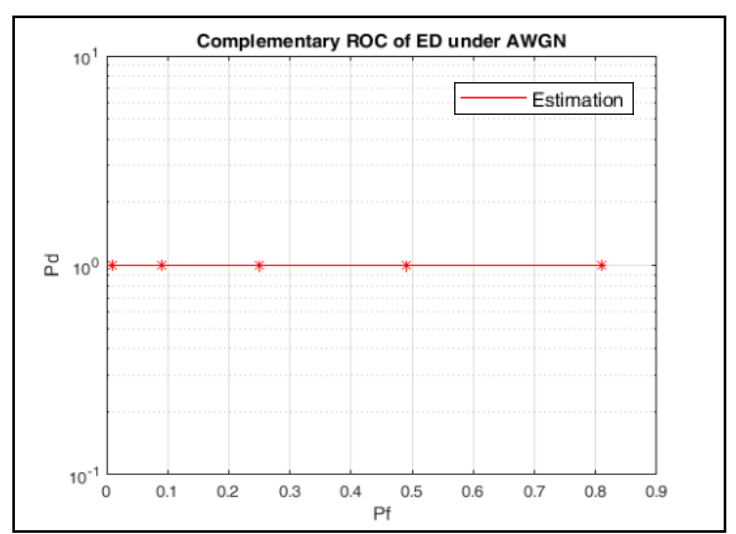

Figure 13: The complementary ROC of energy detection under AWGN for Pf vs Pd according test no. M2C3. 


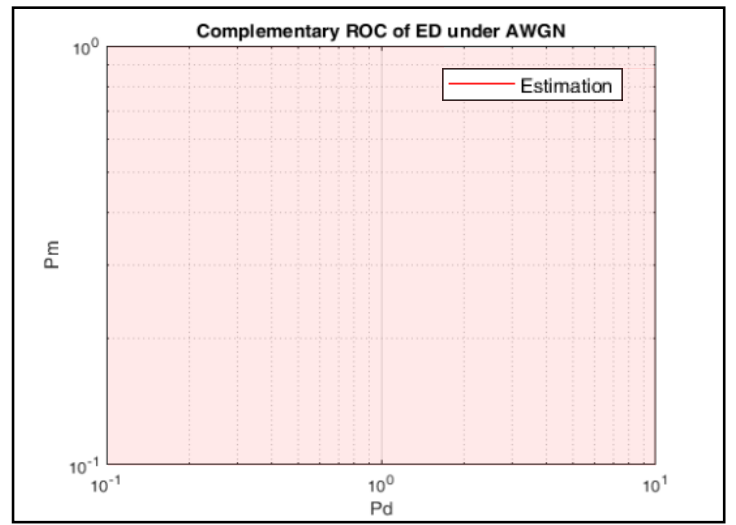

Figure 14: The complementary ROC of energy detection under AWGN for Pd vs Pm according test no. M2C3.

\section{The results of the practical model represents by emulation and prototype device:}

The TVCRW are the abbreviations of the first letters of Television (TV), Cognitive Radio (CR) and WIMAX (W), it is placed in this arrangement to symbolize the work of cognitive radio between the WiMAX system and DTV-T system .This device is designed to test the programming implemented by the Matlab simulation and to make sure that:

1. Achieve the basic goal of this paper to implementation of a common working mechanism with a different priorities for digital communication systems.

2. Achieving the concept of cognitive radio technique in organizing work within a certain frequency.

3. Study the possibility of two different systems working within a same frequency in a sequential manner without interference between them and providing practical solutions in case of loss of connection for the system of minimum priority.

4. Developing the working environment by using the concept of Internet of things to manage the wireless systems remotely from anywhere in the world.

\section{i. The Emulation Program for TVCRW - V1.}

The emulation was implementation by using the Arduino program to verify the validity of the program and used the Proteus ISIS 7 for visual representation this model, figure 15 shows the Arduino programmer and figure 16 shows the device in the emulation environment.

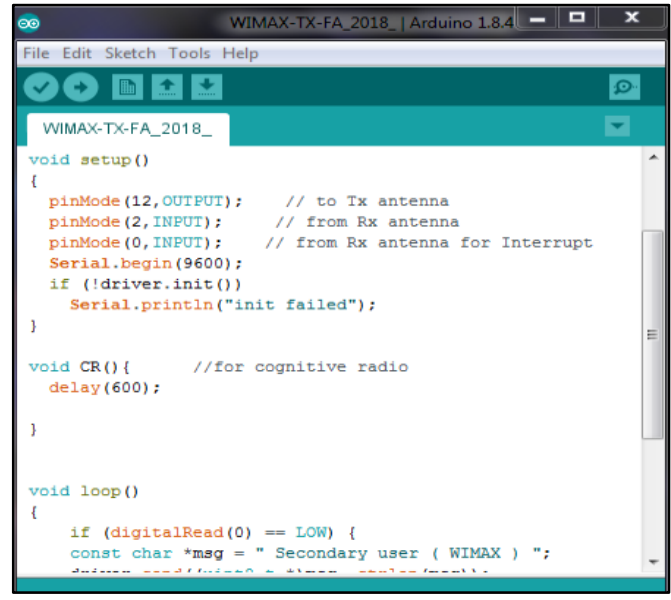

Figure 15: The Arduino programmer.

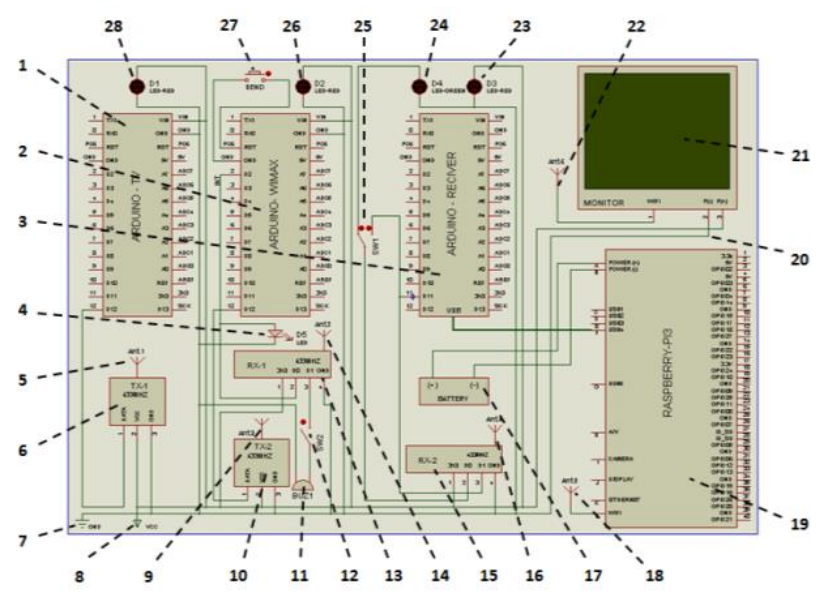

Figure 16: The device in the emulation environment.

The method and mechanism for this device can be explained by figure 16. The digital TV terrestrial is represents the basic system or the primary user and it is controlled by an Arduino controller - TV (1) connected to a Transmitter unit - TV (6) and which in turn are linked to a Transmitter antenna - TV (5). The WiMAX system is the internet system or the secondary user it is represent here by an Arduino controller -WiMAX (2) connected to a Transmitter unit-WiMAX (10) which in turn is connected to a Transmitter antenna- WiMAX (9) .the Receiver antennaCR (14) is connected to a separate Receiver unit-CR (13) .the reception unit (cognitive radio) is very important in the spectrum sensing to determine the frequency if it was concerned by primary user of the highest priority or not, and then sent this result to arduino controller -WiMAX to using the frequency by the secondary user or following the instructions of the TVCRW Adapter .

The result of the sensing can be defined by a built-in lighting (4) or an Alarm buzzer that changes according to the status of the channel (11) where the two options are switched by a Key (12) and the start the exploiting for the frequency by secondary user after the achieving all the conditions by clicking on the confirmation Pushbutton (27) that included in the device of WiMAX system. The client device represent here by Arduino controller-CL (3). The three devices (WiMAX, TV broadcasting and the client) were supplied with three independent lamps (lEDs) (23), (26), (28) indicating whether the device is working or not. The client is receives the information from the two systems by 
unidirectional link (download) via a Receiver antenna-CL (16) connected to a Receiver unit-CL (15). The client device is also equipped with a Key (25) which enables the user to choose between the two systems in the form of messages that can be viewed through the LCD Screen attached to the client (21) or via a pulse from custom LED the in the same device (24). The data information is also routed to the screen over the air (18) via the Raspberry-Pi controller (19) also it can be viewed via the Smart phone screen (22) after installation of certain programs such as VNC viewer. The Power supply $5 \mathrm{v}$ DC was connected to the system (7\&8), It was also added another source of energy (Battery) (17) and the parts were connected by electrical wiring (20).

\section{ii. The Prototype Model for TVCRW - Vl.}

To implement this device was used the several programs they are as follows: used the Arduino Programmer +7 Libraries to write the arduino program, used the Raspbianjessie +4 Libraries to programming the raspberry-Pi B, used the SD Formatter to format the memory card for raspberry-Pi B system, used the Win32diskimager to program interface to burn the program in the SD memory and used the VNC viewer to monitoring. and also used the several hardware components they are as follows: used the Arduino-Nano to control unit in the practical module, used the Raspberry-Pi B to assistant the control unit and used the Smart Phone to communicate with the system from anywhere, figure 17 shows the TVCRW during the manufacturing and figure 18 shows the TVCRW during the testing and inspection., can be extracted the mechanism from the flowchart in section 3 in this paper.

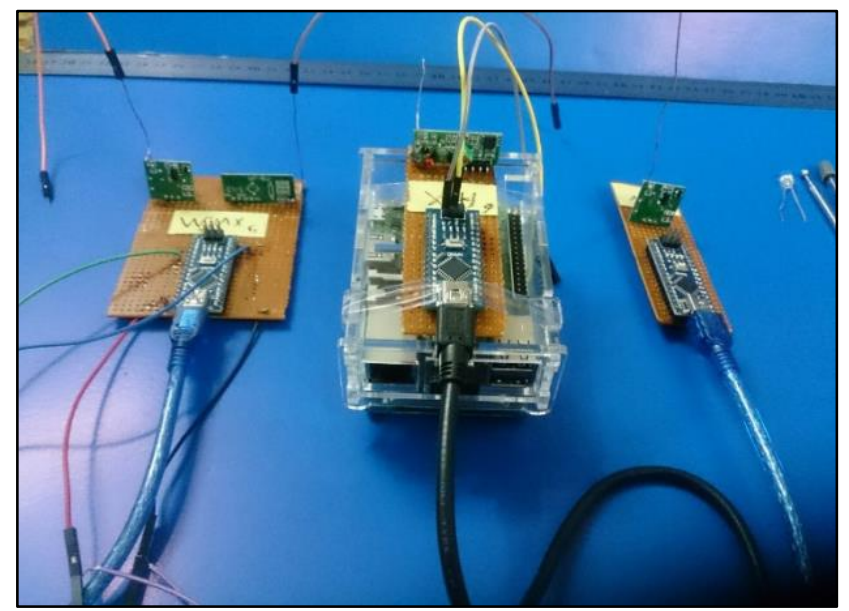

Figure 17: The TVCRW during the manufacturing.

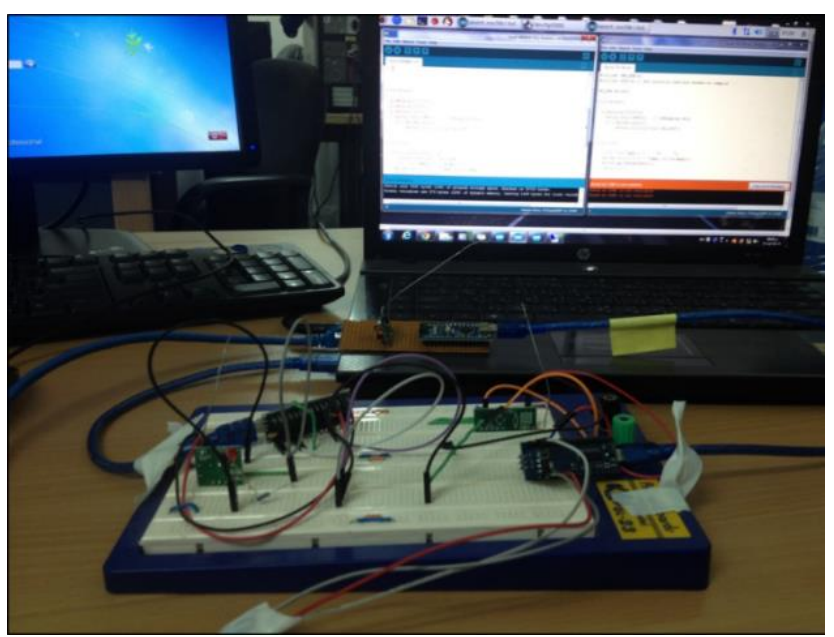

Figure 18: The TVCRW during the testing and inspection.

\section{iii. The final form of Model for TVCRW - VI.}

The prototype module (TVCRW) is manufactured from recycled materials and simple components.

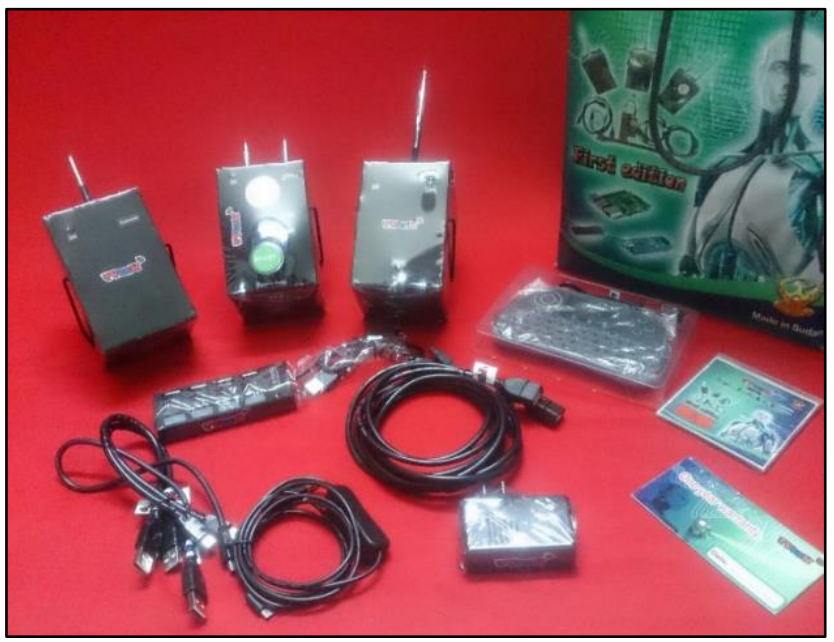

Figure 19: The final form for the TVCRW-v1 prototype and its accessories.

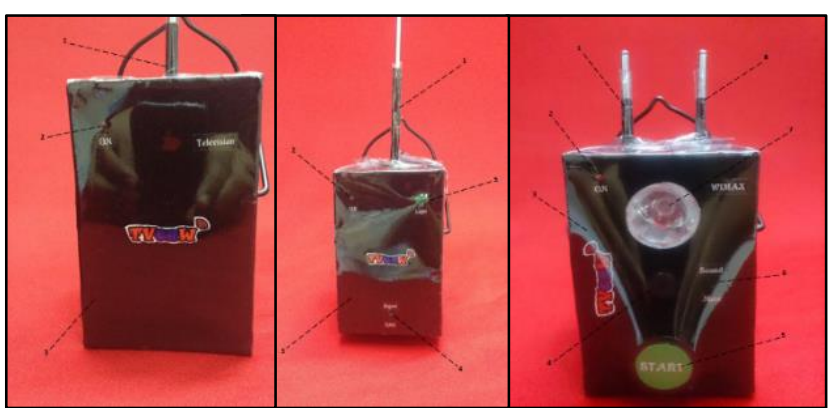

(a) (PU).

(b) (CPE).

(c) $(\mathrm{SU})$.

Figure 20: The three basic (a, b and c) parts of TVCRW system from the front side.

$\mathrm{PU}=$ primary user equipment.

$\mathrm{CPE}=$ customer premises equipment.

$\mathrm{SU}=$ secondary user equipment. 
The figure 20 (a) represents the primary user equipment (PU), so that the number 1 shows transmitter antenna, number 2 shows the LED to indicate the status of the device (on or off), number 3 shows the external body of the device. And the figure 20 (b) represents the customer premises equipment (CPE), so that the number 1 shows the receiver antenna, number 2 shows the LED to indicate the status of the device (on or off), number 3 shows the external body of the device, number 4 shows the key to choose the view of the acquisition result, number 5 shows the LED used to display the results via expressive optical pulses. And the figure 20 (c) represents the secondary user equipment (SU), so that the number 1 shows the transmitter antenna, number 2 shows the LED to indicate the status of the device (on or off), number 3 shows the external body of the device, number 4 shows the buzzer used to the show the states of the spectrum frequency via the expressive sound pulses, number 5 shows the push button switch to activate the acquisition, number 6 shows the buzzer control switch, number 7 shows the LED used to the show the states of the spectrum frequency via the expressive optical pulses, number 8 shows the receiver antenna connected with the cognitive radio unit.

\section{E. Flowchart for TVCRW-v1 Model}

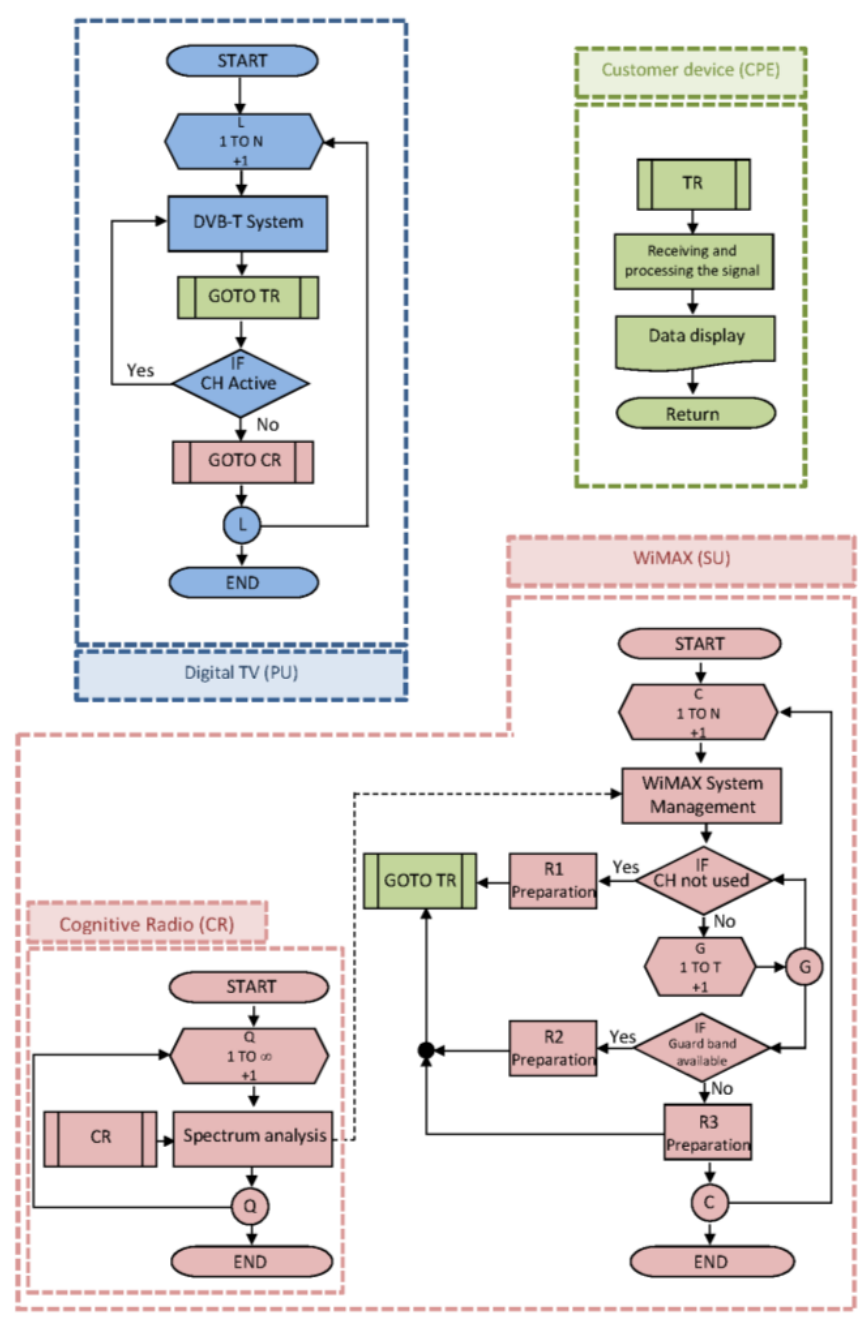

\section{CONCLUSION}

This paper investigates how to design a model that allows using the same radio spectrum from two different systems under a mechanism called by the cognitive radio network. Where extract from the study of results the possibility of providing a common working environment based on priorities so the frequencies trading are done according to the outputs of the units of sensing and perception see figures 3 to 8 .also notes where the greater the probability of false alarm the greater the probability of detection and the opposite is true, see figure 11.and the greater the probability of detection the decrease the probability of missing and the opposite is true, see figure 12 . The increase of the signal to noise ratio (SNR) lead to supports of the probability distributions under the null and alternative hypotheses become more disjoint. Therefore, for a given false alarm probability, the probability of detection increases, see figure 10.

\section{ACKNOWLEDGMENT}

I am very thankful to everyone who supported me. And would like to take the opportunity to thank people who guided and supported me during this work. I am very grateful to my parents who have always given me their unconditional caring and support, and to extend thanks to many other colleagues who helped in reviewing the WiMAX system performance. Last but not the least, I would like to thank my friends and to those invisible hands that have helped me in this project. I can't thank you enough.

\section{REFERENCES}

[1] Shabnam Sodagari, APRIL 2018, Enhanced Spectrum Sharing and Cognitive Radio Using Asynchronous Primary and Secondary Users, IEEE communications letters, vol. 22, no. 4.

[2] G. Mastorakis, 2011, TV White Spaces exploitation utilizing a Cognitive Radio system based on DVB-H, IEEE.

[3] Dr.techn. Markus Rupp, February 2007, Implementation of a WiMAX simulator in Simulink.

[4] Mohammad Azizul Hasan, June 2007, Performance Evaluation of WiMAX/IEEE 802.16 OFDM Physical

[5] Layer.

[6] E.Pietrosemoli, December 2013, TV white spaces a pragmatic approach.

[7] Professor Kwang, 2009, Cognitive Radio Networks

[8] Stephen J. Shellhammer, May 2009, Technical Challenges for Cognitive Radio in the TV White Space Spectrum, IEEE.

[9] Santa Rahman, January 2013, Comparative Study between Wireless Regional Area Network (IEEE Standard 802.22) and WiMAX and Coverage Planning of a Wireless Regional Area Network Using Cognitive Radio Technology, International Journal of Recent Technology and Engineering (IJRTE) ISSN: 2277-3878.

[10] Jeffery G. Andrews, 2007, Fundamentals of WIMAX, foreword by Theodore Rappaport, Series Edition.

[11] Naroa Zurutuza, June 2011, Cognitive Radio and TV White Space Communications, MSC in Norwegian University of Science and Technology.G. Mastorakis, 2011, TV White Spaces exploitation utilizing a Cognitive Radio system based on DVB-H, IEEE. 


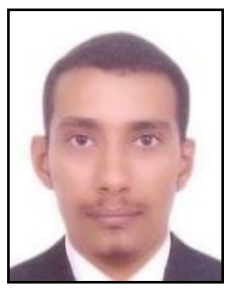

Eng. Albedeir Yaseen Othman currently worked as PhD student, received his MS.C Degree in communication engineering from Sudan University of Science and Technology in 2012. His Many of patents in the field of communications and remote control, His research is focused on wireless communication and embedded systems. Currently he is working for in Dar al-handasah consultants, KSA. He is Director of management in technology development organization (TDO), and one of the most important founders.albedeir1985@yahoo.com.

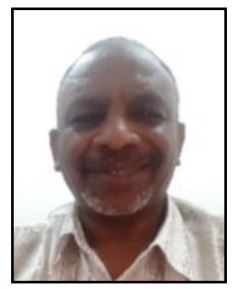

Dr.Khaled Hamed Bilal currently worked as professor, Faculty of Engineering, University of Science \& Technology, in Sudan. After received his $\mathrm{PhD}$ degree in 2008, his worked as head of communication Engineering Department. He supervised numerous post graduate and undergraduate students in their research projects. His have contributed positively in the development of some university in Sudan and its faculties. His research interest includes communication and computer engineering. khalidhamidk9@gmail.com.

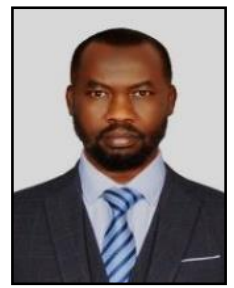

Dr. Mohammed Elmaleeh currently worked as associate professor, Tabuk University, KSA. After received his $\mathrm{PhD}$ degree in 2009, his worked as assistant and associate professor for a couple of years in Sudan. He supervised numerous post graduate and undergraduate students in their research projects. He was assigned as a reviewer and track chair for several IEEE conferences and international journals. His research interest includes embedded systems, communication and computer engineering. maleeho@ hotmail.com. 